While the removal of hydrogen chloride from such molecules is clearly an endothermic process there can be no doubt at all but that these induced pyrolyses are chain reactions. Although there is a superflcial resemblance to the well-known iodine-catalysed decompositions of aliphatic ethers, ketones, etc., the two types of induced reaction must be quite different. The effectiveness of iodine in the ether and ketone reactions is to be related ${ }^{2}$ to its facile dissociation to the atomic form ; iodine is much superior, therefore, to chlorine or bromine, whereas, with the ethylene dichloride decomposition, such is not the case. Nevertheless, as with iodine-catalysed decompositions, the activation energies of the induced pyrolyses of ethylene dichloride are much less than that for the uncatalysed reaction. It is of interest that Sherman, Quimby and Sutherland ${ }^{3}$ have already predicted, on the basis of calculated activation energies, that the decomposition of ethylene dichloride should be catalysed by halogens, but their method can offer no explanation as to the remarkable dependence of these induced reactions on molecular structure (see above). In any of these induced reactions on molecular structure (see above). In any
event, it is still not certain that these reactions are of the radicalevent, it is still not certain that these reactions are of the radical-
chain type postulated by them, and a number of the experimental facts chain type postulated by them, and a number oc the exp.

The investigation of the kinetics, mechanism and scope of these The investigation of the kinetics, mechanism and scope of these
induced reactions is at present being undertaken. The industrial induced reactions is at present being undertaken. The industrial
utilization of the facts presented here is covered by British Patents utilization of the facts presented here is covered by British Patents
573,532 and 573,559 , but prior publication has been prevented by war-time secrecy restrictions.

I am greatly indebted to Dr. M. Mugdan, of the Distillers' Co., for the diblish of that Company for financial assistance and permission to publish this letter.
D. H. R. BARTON.

Imperial College of Science and Technology, London, S.W.7.

${ }^{1}$ Kaplan, Grishin and Skvortsova, J. Gen. Chem. (U.S.S.R.), 7, 538 (1937).

${ }_{3}^{2}$ Hinshelwood, "The Kinetics of Chemical Change", 244.

J. Chem. Phys., 4, 732 (1936).

\section{Tetrapositive Nickel as Alkali Nickel Periodates}

THE formation of the black oxides of nickel as well as of the so-called nickelites suggests that nickel can occur in a state of valency higher have been assumed in these compounds, though they have seldom been obtained in a pure state with definite composition. Some apparently double molybdates of quadrivalent nickel with alkali and alkalin earth metals have been described and studied by Hall'. We have shown that these fine purple black crystalline compounds should be regarded as salts of complex heteropoly molybdic acid with tetrapositive nickel as the central atom. Measurement of the magnetic susceptibility of these complex nickelic molybdates in our Laboratory ${ }^{2}$ has shown that they are all diamagnetic. This proves that the tetrapositive nickel atom in these serves as the centre of a penetration complex with octahedral $d^{2} s p^{3}$ hybrid bonds resembling the tripositive cobalt in cobaltic complexes. A consideration of the fact that tetrapositive nickel and tripositive

of electrons readily accounts for it.

We have now been able to prepare specimens of sodium and potassium nickel periodates with nickel in the tetrapositive state. These were prepared by oxidizing a mixture of nickel sulphate solution and the corresponding alkali periodate at the boiling temperature with sodium or potassium persulphate as the case may be. The substances form dark purple, almost black, insoluble fine crystals with metallic lustre and have a tendency to adhere to the walls of the beaker in the form of a bright mirror. Their composition is represented by the formula, $\mathrm{Na}(\mathrm{K}) \mathrm{NiIO}_{6} \cdot x \mathrm{H}_{2} \mathrm{O}$, apparently a mixed salt of orthoperiodic acid, $\mathrm{H}_{5} \mathrm{IO}_{6}$, the hydrogen atoms of which are replaced by periodic acid, $\mathrm{H}_{5} 1 \mathrm{O}_{6}$, the hydrivalent nickel and one alkali metal atom. Measurement of the magnetic susceptibility, however, shows that the substance should the magnetic susceptibility , however, shows that the substance should not be represented as an ionic compound by the simple formula as given above. A quadrivalent nickel ion in the normal state should
give a high moment of $4.9 \mathrm{Bohr}$ due to four unpaired electrons. The give a high moment of 4.9 Bohr due to four unpaired electrons. The compounds, however, give rather a low moment of only 1.2 Bohr approximately On the other hand, a diamagnetic susceptibility
would, as in the case of the above-mentioned heteropoly nickelic would, as in the case of the above-mentioned heteropoly nickelic molybdates, indicate the formation of hybrid $d^{2} s p^{2}$ bonds with an octahedral configuration of oxygen atoms around the tetrapositive
nickel atom in the crystal. A quadrivalent nickel, like its higher nickel atom in the crystal. A quadrivalent nickel, like ty higher homologues quadrivalent palladium and platinum, is likely to have a co-ordination number of six. The measured moment seems to favour the latter view, assuming a partial dissociation of the substance
into $\mathrm{KIO}$ and $\mathrm{NiO}$, due to its temperature instability, which would into $\mathrm{KIO}_{4}$ and $\mathrm{NiO}_{2}$, due to its temperature instability, which would
account for the observed paramagnetic susceptibility giving a low

account for the

On this basis, the possible structure of these alkali nickel periodates in the crystalline state may be represented as follows. Each nickel, jodine or potassium atom is surrounded octahedrally by six oxygen atoms, and each oxygen is bound to one nickel, one iodine and on potassium atom showing a co-ordination number of three. The bond between oxygen and nickel, as well as those between oxygen and iodine, are predominantly co-valent, due to strong polarizing power of the highly positive nickel (quadrivalent) and iodine (heptavalent) atoms, while those between potassium and oxygen are purely ionic. The $\mathrm{Na}(\mathrm{K})\left[\mathrm{Niv}\left(\mathrm{IO}_{*}\right)\right]$.

P. RAY.

Inorganic Chemistry Laboratory,

University College of Science, Jan. 21.

${ }^{1}$ Hall, J. Amer. Chem. Soc., 29, 692 (1907).

2 Rây and Bhaduri, Indian Science Congress, 1945.

\section{Superheating and 'Bumping' of Organic Liquids}

THE superheating and 'bumping' of some liquids is well known to organic chemists, but I wonder if the dangers of superheating are sufficiently widely realized. The following incident occurred recently. Preparing butyl bromide by a usual method, I heated a mixture of $n$-butyl alcohol (90 gm.), hydrobromic acid $(250 \mathrm{gm}$.) and sulphuric acid (110 gm.) under reflux in a $500 \mathrm{ml}$. flask on an electric hot-plate. By an oversight, I omitted the usual piece of porous pot. The tempera. ture rose steadily until liquid was distilling back from the reflux condenser at the rate of about three drops a second, yet the surface of the liquid in the flask remained quiescent. Suspecting superheating
and possible trouble, I switched off the hot-plate and let the flask and contents cool until the liquid was distilling back from the reflux at a rate of not more than one drop every ten seconds; then I reflux at a rate of not more than one drop every ten seconds; then I
lifted the condenser and dropped in a piece of porous pot. The result was astonishing, the contents of the flask being thrown with consider. was astonishing, the contents of the flask being thrown with consider-
able violence against a wall three feet away. Fortunately, I escaped able violence against a wall three feet away. Fortunately, I escaped
with nothing more serious than the loss of my preparation, some with nothing more serious than the loss of my preparation, some
broken apparatus and an afternoon wasted in 'mopping up operations'; broken apparatus and an afternoon wasted in 'mopping up operations';
but the dangers of similar accidents with such corrosive mixtures but the dangers of similar accidents with such corrosive mixtures
in crowded school or university laboratories are too obvious to need in crowded

I believe that the use of the hot-plate in this instance helped to promote superheating; but I have found that even with the addition of sand or porous pot, this particular mixture is inclined to 'bump' badly during the early stages of the preparation and needs careful watching. Students will be well advised, if ever they have reason to suspect superheating, to turn off the heat at once and make sure that the liquid they have been heating has, in fact, cooled to below the normal boiling point before they add porous pot or proceed further
with the experiment. This simple precaution seems obvious, but it with the experiment. This simple precaution

"Inglemere",

V. G. W. HARRISON.

Dagden Road,

Guilfford.

Is Carotene a Provitamin A, or has it Biological Activity per se?

IT is generally acknowledged that the carotenes are 'provitamins', but there is no clear deflnition of the term 'provitamin' in the literature; the general view seems to be that it is a compound which is not a vitamin itself but after minor changes is transformed into one. The organism may be incapable of carrying out this transformation (D-provitamins), or it may be able to do so (A-provitamins). The term 'provitamin A' was first used by Harris', while Moore ${ }^{2,3}$ called carotene a precursor of vitamin A. The opinion of these and all subsequent authors seems to be based only upon Moore's experiments on storage of vitamin A. As in the rat the minimal doses necessary to cause storage are about ten times those giving optimal biological
effect, 4 , the deposition and the biological action take place at widely separated dose-levels. Further, the former takes place in the liver, while the latter takes place all over the organism (surface epithelium, nerve cells, retina) where it cannot be followed by chemical analyses. that the carotenes do not possess biological A-action per se because they are stored in the form of vitamin A.

they are stored in the form of vitamin A.
Moore $^{7}$ writes: "Both carotin and the classical 'vitamin' may independently possess the same physiological action-" but this possibility is not mentioned in his later papers. Palmer ${ }^{8}$ writes of possibility is not mentioned in his later papers. Palmer ${ }^{8}$ writes of certain $A$-active artiffial compounds:
this biological activity is due to the fact that they are changed to the this biological activity is due to the fact that they are changed to the classical vitamin $\mathrm{A}$ in the body or possess physiological activity per $8 e^{\prime}$,
but that the same can be said of the carotenes themselves is not but that the

The optimal requirement for vitamin $A$ in mammals is 15-25 I.c. or 5-8 $\gamma$ per $\mathrm{kgm}$. a day and for $\beta$-carotene 30-50 I.U., or 18-30 $\gamma^{4}$. The amount of vitamin $A$ (in $\gamma$ ) stored as a percentage of the A-actire substance (in $\gamma$ ) given is $15-25$ for vitamin $A$ and $1.2-3.8$ for $\beta$ carotene in the rat . In the hen the requirement is 1.0. $33-66 \gamma$ per kgm. a day for vitamin A and 100-200 1. . Or $75-150 \gamma$ that of vitamin $\mathrm{A}$ and in mammals four times that of vitamin $\mathrm{A}$. As the hen's requirement is about ten times that of a mammal, it suggests that small birds which seem to have a vitamin A requirement per $\mathrm{kgm}$. many times greater than the hen ${ }^{11},{ }^{12}$ might show yet greater differences and be experimental subjects worth attention.

$\alpha$ - and $\gamma$-carotene ${ }^{13}$, cryptoxanthin $^{14}$ and $\beta$-oxycarotene ${ }^{15}$ are about half as active as $\beta$-carotene, which is in good agreement with the fact that each molecule of these substances can form one molecule of vitamin A, while $\beta$-carotene can form two. From the data in the literature ${ }^{16,17,14}$, it is possible to calculate the storage percentage of these carotenoids as $4-8$ per cent for $\beta$-carotene and a third to a half of this for the others with the exception of $\beta$-oxycarotene, which gives 0.1 per cent ${ }^{14}$. In hens the activity and storage of cryptoxanthin is known from experiments with yellow corn" ${ }^{8,10} ; 90 \gamma$ of $\beta$-carotene showed the same biological action as $36 \gamma$ cryptoxanthin plus $18 \gamma$ of $\beta$-carotene, and $2 \cdot 2 \mathrm{mgm}$. cryptoxanthin plus $\beta$-carotene (with two So the cryptoxanthin) caused the same storage as carotene in the hen-compare $90 \gamma$ and $36+18 \gamma$-and the storage capacity for cryptoxanthin is even higher than that for $\beta$-carotene. As in the hen, the biological activity of vitamin A per $\gamma$ is about twice that of $\beta$-carotene, the activity of vitamin $A$ and cryptoxanthin must be of the same order of magnitude. As cryptoxanthin
produce, at the most, half its weight of vitamin A, this means that it produce, at the most, half its weight of vitamin A,

must possess vitamin a A a ctivity per ee the rat and the hen. Perhaps cryptoxanthin acts as vitamin $\mathbf{A}$ per $8 e$ in the hen and not in the rat. 